\title{
Correspondence
}

\author{
New joints, same moves: \\ the ossification of community psychiatry
}

Community psychiatry is at a crossroads and Peter Tyrer's critique $^{1}$ is timely and welcome. Although problems in community care were developing before the economic downturn, the present financial climate has sharpened the issues and makes finding a solution more pressing than ever.

There have been significant investments in community care over recent years. Mandated by central policy this has resulted in an increased subspecialism, with the development of new community teams focusing on early intervention, crisis work and assertive outreach. The clinical effectiveness of these new teams is hotly debated but an undeniable consequence has been to diminish continuity of care and to create a more fragmented service, with multiple interfaces, each timeconsuming and risky to negotiate. The residual community teams have been overwhelmed by the volume and complexity of demand, over-burdened by bureaucracy, and sometimes treated as little more than the handmaidens to specialist services.

The newly formed specialist community teams have had the advantage of defining their place in the system; facilitating the delivery of evidence-based interventions and fidelity to models of care. Tyrer argues for the re-establishment of 'completely comprehensive' teams, but the tensions that have challenged community teams will survive a structural reconfiguration. Community teams need to deliver care which is individually formulated but not at the expense of evidencebased treatments. Care needs to be responsive and holistic but this approach has to be balanced with the need to deliver planned treatments. How can these tensions be resolved?

Community teams cannot and should not provide every intervention for patients under their care - to do so creates dependency and a new form of institutionalisation. We need to move from providing holistic care to facilitating holistic care, working with the community, not just in it. We need to establish and formalise robust pathways that facilitate timely access to outside agencies, where patients can receive support for issues such as housing and benefits advice. We need to define the boundaries of unplanned, responsive care delivered by the community team. Should this be available to all patients or restricted to those most disabled by their illness? What unplanned interventions are the task of the team and which sit with other external providers? How do we create systems to deliver a flexible and timely response to need while retaining capacity for clinicians to deliver planned interventions?

Finally, we need to deconstruct care coordination, retaining the important clinical functions but removing the unnecessary bureaucracy that adds little to patient care. On a practical level, these are the issues which challenge community teams and they need to be addressed along with any structural reorganisation.

1 Tyrer P. A solution to the ossification of community psychiatry. Psychiatrist 2013; 37: 336-9.

Tim Oakley is consultant psychiatrist, St George's Park, Morpeth, Northumberland, UK, email: tim.oakley@ntw.nhs.uk

doi: $10.1192 / \mathrm{pb} .38 .1 .45$

\section{Is it too late for a solution to the ossification of community psychiatry?}

Professor Tyrer's article ${ }^{1}$ has been such a comforting read, as many of his thoughts expressed will resonate with views of many average, hard-working, catchment area psychiatrists. The fragmentation of psychiatric services has already occurred and is likely to continue in the immediate few years. Psychiatrists as professionals have difficulties sustaining therapeutic relationships (the anchor in any healing process) for any decent length of time with patients who often are traumatised, ill and vulnerable.

Professor Tyrer's solution lives up to the College's motto 'Let Wisdom Guide' and makes a lot of sense to the dying-out breed of catchment area psychiatrists, but has it come too late? The 'product champions' of different service models are likely to rise up in defence of their brands and the new lot of fragmented-care psychiatrists may have visions of themselves as super-specialists, and so may see catchment area psychiatrists as belonging to a bygone era. But maybe, in this new era of reflection, we should all spare some time and reflect on our College's motto and Professor Tyrer's words of wisdom.

1 Tyrer P. A solution to the ossification of community psychiatry. Psychiatrist 2013; 37: 336-9.

Thakor Mistry is consultant psychiatrist, Black Country Partnership NHS Foundation Trust, West Bromwich, UK, email: thakor.mistry@bcpft.nhs.uk doi: $10.1192 / p b .38 .1 .45 a$

\section{Where is the evidence for incorporating early intervention treatment into the CMHT?}

Although it is gratifying to be regarded as the 'best of the staff' by someone as esteemed as Professor Peter Tyrer, I take issue with the suggestion that early intervention teams (EITs) should be broken up and their functions incorporated within a flexible community mental health team (CMHT). ${ }^{1}$ Following the principle of 'Let Wisdom Guide', one would like to see the evidence before taking such a step. For while it may be true that assertive outreach teams and other innovations in Britain proved disappointing for some of the reasons outlined in the article, this is not the case for early intervention. For example, there is evidence that EITs reduce hospital admission compared with $\mathrm{CMHTs}^{2}$ and that once patients are transferred back to CMHTs, the admission rate goes up again. $^{3}$

If we have a service model of proven effectiveness, particularly in reducing demand on the most expensive elements of mental healthcare (in-patient beds), such as EITs, why switch to an unproven service model? One can make a tentative case that the superior outcomes are due to 'better skilled' EIT staff or to the extra resources these teams have - which the McCrone paper shows pays for itself by reducing demand ${ }^{3}$ - but a wise approach suggests waiting for evidence of effectiveness of these CMHTs with EIT 
functionality before ploughing ahead and dismantling an evidence-based superior service.

\section{Declaration of interest}

A.S.H. is a consultant in an early intervention team.

1 Tyrer P. A solution to the ossification of community psychiatry. Psychiatrist 2013; 37: 336-9.

2 Gafoor R, Nitsch D, McCrone P, Craig TKJ, Garety PA, Power P, et al. Effect of early intervention on 5-year outcome in non-affective psychosis. Br J Psychiatry 2010; 196: 372-6.

3 McCrone P, Craig TKJ, Power P, Garety PA. Cost-effectiveness of an early intervention service for people with psychosis. Br J Psychiatry 2010; 196: 377-82.

Ahmed Samei Huda is a consultant psychiatrist, Tameside and Glossop Early Intervention Team/South Sector CMHT, Pennine Care NHS Foundation Trust, Dukinfield, UK, email: ahmed.huda@nhs.net doi: $10.1192 / p b .38 .1 .45 b$

\section{Assertive community treatment: keeping what works}

The excellent editorial by Rosen et al highlights the dilution of assertive community treatment (ACT) research in European settings, leading to a failure to demonstrate reductions in bed use in efficacy studies. Effectiveness studies in the UK have shown that ACT leads to reductions in bed use. ${ }^{2,3}$ Furthermore, our experience of 93 patients followed up for a mean of 6.5 years after starting ACT showed a reduction from a mean of 72 days per year prior to ACT to 44 days per year during ACT $(P=0.002)$. Repeated measures using the Dartmouth Assertive Outreach Fidelity Scale ${ }^{4}$ demonstrated the team had high fidelity to the assertive outreach model.

The evidence supports the importance of trying to incorporate the effective components of ACT into new services. Despite these benefits, ACT teams continue to be dismantled.

1 Rosen A, Killaspy H, Harvey C. Specialisation and marginalisation: how the assertive community treatment debate affects individuals with complex mental health needs. Psychiatrist 2013; 37: 345-8.

2 Mortimer AM, Shepherd CJ, Fadahunsi A, Jones A, Kumar P, Gangaram P. Assertive outreach: mirror-image study with contemporaneous controls. Psychiatrist 2012; 36: 245-8.

3 Rana T, Commander M. Long-term follow-up of individuals on assertive outreach teams. Psychiatrist 2010; 34: 88-91.

4 Teague GB, Bond GR, Drake RE. Program fidelity in assertive community treatment: development and use of a measure. Am J Orthopsychiatry 1998: 68: 216-32.

Loopinder Sood is associate specialist in community psychiatry and Andy Owen is consultant in community psychiatry, Coventry and Warwickshire NHS Partnership Trust, St Michael's Hospital, Warwick, UK, email: loopinder.sood@covwarkpt.nhs.uk

doi: $10.1192 / \mathrm{pb} .38 .1 .46$

\section{Looking at vanishing treatment effect in Europe}

The article by Rosen et al is a thought-provoking piece on the perception of the effectiveness of assertive community treatment in Europe. I write as a psychiatrist who has worked within assertive community teams (ACTs) on both sides of the Atlantic (the UK and Canada). Although the economic downturn and cutbacks are equally shared in both UK and Canadian health systems, the investment in specialist mental health programmes including ACT continues to gather pace in Canada due to the robust and demonstrable effectiveness of the teams. In Canada, there is a stronger fidelity to the original ACT model and a shift from the paternalistic to a more collaborative relationship with patients. There is greater emphasis on relationship building through varieties of psychosocial strategies, easier access to specialist and subsidised housing facilities and vocational opportunities.

The ACT service delivery in Canada is a true representation of the standard originally described in 1973 after 20 years of field testing in the USA. ${ }^{2}$ It includes peer support workers who have life experience and can provide expertise that professional training cannot replicate. The peer support workers are fully integrated team members functioning in the team's generalist role. There is a 24-hour on-call service and treatment intervention is intensive with two or three face-to-face visits daily.

Undoubtedly, ACT is a clinically effective approach to managing the care of severely mentally ill people in the community. If aimed at the right patient population and when the model of care is fully adhered to, ACT can substantially reduce the costs of hospital care while improving the outcome and patient satisfaction. ${ }^{3}$

1 Rosen A, Killaspy H, Harvey C. Specialisation and marginalisation: how the assertive community treatment debate affects individuals with complex mental health needs. Psychiatrist 2013; 37: 345-8.

2 Marx AJ, Test MA, Stein LI. Extrahospital management of severe menta illness: feasibility and effects of social functioning. Arch Gen Psychiatry 1973: 29: 505-11.

3 Test MA, Stein LI. Alternative to mental hospital treatment. I. Conceptual model, treatment program, and clinical evaluation. Arch Gen Psychiatry 1980; 37: 392-7.

Stephen A. Ogunremi is consultant psychiatrist, Fraser Health Authority, Vancouver, Canada, email: Stephen.Ogunremi@fraserhealth.ca

doi: 10.1192/pb.38.1.46a

\section{Quality of risk assessment prior to suicide and homicide}

We are grateful to Large \& Nielssen ${ }^{1}$ for their interest in the report from our pilot study, ${ }^{2}$ but think they may have misunderstood its main purpose. We wished to investigate the 'low risk paradox' - the fact that in the National Confidential Inquiry data, risk is nearly always reported as low prior to suicide and homicide. ${ }^{3}$

We agree that risk assessment in people who do not die is of interest but our study was not set up to investigate this. Equally, contradictory risk factors are of interest but our focus was on the risk assessment process itself, not on a tally of risk factors or whether they were the 'right' ones. Last, our study was not an investigation of the predictive utility of risk assessment. We are familiar with the low base rate problem and have written about this elsewhere. ${ }^{4}$

We would wholeheartedly agree with Large \& Nielssen's suggestion that assessments should be compassionate, ethical and needs-focused. However, we would take issue with their 